\title{
Spontaneous hyphaema associated with anterior uveitis
}

Department of Ophthalmology, Massachusetts Eye and Ear Infirmary and Harvard Medical School, Boston, USA

D S Fong

M B Raizman *

$\star$ Current address: New England Eye Center, Tufts University School of Medicine, Boston, MA, USA

Correspondence to: Donald S Fong, MD, Department of Ophthalmology Massachusetts Eye and Ear Infirmary, 243 Charles Street, Boston, MA 02114, USA. Accepted for publication 31 May 1993
Donald S Fong, Michael B Raizman

\begin{abstract}
Few reports have described hyphaema in association with anterior uveitis. Five cases of anterior chamber haemorrhage are reported in patients with five different anterior uveitic entities: Reiter's syndrome, juvenile chronic arthritis, ankylosing spondylitis, idiopathic anterior uveitis, and herpes simplex. Hyphaema has been reported in association with idiopathic non-granulomatous anterior uveitis, but not with the other four entities. In three cases, iris rubeosis was present. In two cases the patients were taking non-steroidal anti-inflammatory agents. The hyphaemas occurred at times of heightened inflammation and resolved spontaneously without complication in all but one case, a boy with idiopathic uveitis who required surgery to remove the blood. The clinical outcome of these cases provides evidence that conservative medical management is usually sufficient.
\end{abstract}

(Br F Ophthalmol 1993; 77: 635-638)

Anterior chamber haemorrhage is most often associated with trauma. Although spontaneous hyphaema occurs in association with a number of ocular abnormalities (see Table 1), its occurrence in eyes with anterior uveitis has not been well documented. We report five cases of hyphaema in eyes with uveitis associated with Reiter's syndrome, juvenile chronic arthritis, ankylosing spondylitis, idiopathic anterior uveitis, and herpes simplex. Of the five cases, two patients had iris rubeosis, one was taking non-steroidal anti-inflammatory agents (NSAIAs), and one patient had rubeosis and history of NSAIA use. Although hyphaema has been reported with idiopathic anterior inflammation, associations with the one ophthalmic and the three other systemic diseases have not been made previously. In addition to describing these associations, the current report provides evidence that there are

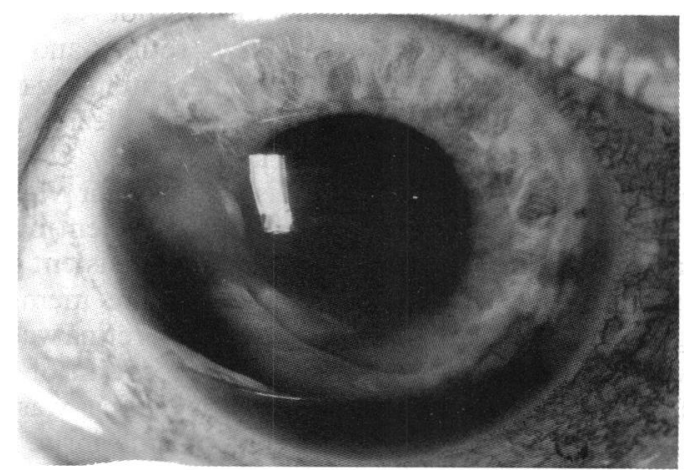

few hyphaema related complications that require more than medical management.

\section{Case reports}

CASE 1

A 44-year-old man presented to the emergency ward complaining of 6 days of photophobia, pain, and redness in his right eye. His medical history was notable for hypertension and a diagnosis of Reiter's syndrome, made 12 years ago when he developed iritis, sterile urethritis, and arthritis of the phalangeal joints. During the past 12 years, he had numerous episodes of chronic non-granulomatous uveitis in both eyes. Each episode responded well to topical steroids and cycloplegia.

Corrected visual acuity measured $6 / 21$ in the right eye and $6 / 9$ in the left eye. The right pupil was $5 \mathrm{~mm}$, round, and poorly reactive to light. The left pupillary responses were normal. No afferent pupillary defect was noted. Extraocular movements were full. Slit-lamp biomicroscopy of the right eye showed moderate conjunctival hyperaemia and a clear cornea. The anterior chamber contained a hyphaema, massive fibrin reaction, and many red and white blood cells (Fig 1). The lens was clear. The anterior segment of the left eye was normal. Intraocular pressure measured 22 and $20 \mathrm{~mm} \mathrm{Hg}$ in the right and left eye, respectively. Few cells were seen in the vitreous of the right eye. Both fundi appeared normal. The blood was HLA-B27 positive. Syphilis serologies were negative.

The patient was treated with oral and topical corticosteroids and topical scopolamine. The hyphaema resolved over the next 2 weeks followed by a resolution of active inflammation. Dilated vessels were then noted on the right pupillary margins at the 10 and 2 o'clock positions. Thes: vessels were neither circumferential nor radial, and were considered rubeotic. Fluorescein angiography of the fundus showed no abnormalities.

CASE 2

A 41-year-old woman was referred to the immunology service for treatment of recurrent inflammation associated with juvenile chronic arthritis. She had recurrent ocular inflammation in the right eye approximately twice yearly since age 1 year, requiring treatment with topical steroids and cycloplegic agents. At times she required systemic steroids and topical timolol to control the inflammation and the elevated intraocular pressure. Four years before our evaluation 
of her, she underwent cataract extraction with sphincterotomies in the right eye. On referral, she reported that her visual acuity had been reduced for 2 weeks in the right eye. She took naproxen (Naprosyn) $250 \mathrm{mg}$ twice a day.

Corrected visual acuity was counting fingers at $10 \mathrm{~cm}$ in the right eye and $6 / 9$ in the left eye. An afferent pupillary defect was present on the right. Extraocular movements were full. Slitlamp biomicroscopy of the right eye showed mild hyperaemia of the limbal conjunctiva. Peripheral band keratopathy at the 3 and 9 o'clock positions was noted on the right cornea. Fine keratic precipitates were noted on the temporal and nasal right cornea, with larger 'mutton fat' deposits inferiorly. A small hyphaema, and moderate cells and flare were seen. Iris rubeosis was present at the 6 and 10 o'clock positions. The posterior capsule was clear. The vitreous contained many cells, including red blood cells and debris. Intraocular pressure measured $17 \mathrm{~mm} \mathrm{Hg}$ on the right and $19 \mathrm{~mm} \mathrm{Hg}$ on the left. The right fundus was not visible. Examination of the anterior and posterior segments of the left eye was unremarkable. The patient received topical corticosteroids and scopolamine and experienced a steady decrease in inflammation. When the vitreous cleared cystoid macular oedema but no ischaemic or haemorrhagic retinopathy was seen in the right eye.

CASE 3

A 25-year-old man presented to the immunology service for iritis refractory to hourly topical prednisolone acetate and 1 day of sudden decreased vision in the right eye. On presentation, he was taking systemic sulindac (Clinoril), topical steroids, and cycloplegics. He was a high myope with a diagnosis of ankylosing spondylitis made in 1981, when he presented with iritis and back pain. At that time, laboratory examination was notable for a positive HLA-B27 blood test and radiographs of his lower back were consistent with ankylosing spondylitis.

Vision measured counting fingers at $1 / 3$ metre in the right eye and $6 / 6$ in the left eye. The conjunctiva of the right eye was hyperaemic. Many red blood cells and a $1 \mathrm{~mm}$ hyphaema were seen in the anterior chamber with white blood cells in the vitreous. The lens was clear. Intraocular pressure measured $10 \mathrm{~mm} \mathrm{Hg}$ in the right eye and $16 \mathrm{~mm} \mathrm{Hg}$ in the left eye. Fundus examination showed a thickened right macula. The patient was continued on hourly topical steroids and systemic sulindac. One week later vision improved to $6 / 18$ on the right and $6 / 6$ on the left. Inflammation in the anterior chamber, vitreous, and fundus was significantly reduced.

Two and a half weeks later vision measured $6 / 21$ on the right and $6 / 6$ on the left. The anterior chamber and the vitreous contained a moderate number of cells. Fundus examination showed a temporal rhegmatogenous retinal detachment in the right eye. The patient was admitted, continued on topical steroids, and started on oral steroids. In the operating room, the tear was sealed with cryotherapy and pneumatic retinopexy, using sulphur hexafluoride $\left(\mathrm{SF}_{6}\right)$ gas. One week postoperatively, the patient's vision remained stable, but he developed subretinal fluid inferiorly. Over the next 2 weeks the inferior collection of subretinal fluid decreased.

Five weeks postoperatively he developed new retinal holes and a new superior retinal detachment. A scleral buckle was performed and areas of lattice noted on earlier examination were treated wth cryotherapy. The postoperative course following the buckle was without complication and both topical and oral steroids were tapered over 2 months. Vision improved to $6 / 7 \cdot 5$ in the right eye and $6 / 6$ in the left.

\section{CASE 4}

A 10-year-old boy with recurrent bilateral anterior uveitis since age 5 presented to the immunology service with an asymptomatic hyphaema in the right eye. He was on topical steroids. In 1984 he underwent bilateral cataract extractions with vitrectomies. He also had multiple EDTA scrubs for the removal of band keratopathy. In addition, cutaneous herpes simplex of the lids was noted on two occasions without ocular involvement. A patent ductus arteriosus was repaired at 2 months of age and bilateral hearing loss and problems with motor coordination were present.

Vision measured counting fingers at 1 metre in the right eye and counting fingers at $1 / 3$ metre in the left eye. External examination was normal. Both pupils were minimally reactive to light. Extraocular movements were full. On slit-lamp examination minimal conjunctival hyperaemia and band keratopathy were seen bilaterally. The right anterior chamber contained a $2 \mathrm{~mm}$ hyphaema. The left anterior chamber was normal. Neither fundus examination nor intraocular pressure measurement could be performed because of lack of cooperation. Examination under anaesthesia showed normal intraocular pressures, a few abnormal iris vessels, cystoid macular oedema, and mild optic atrophy in both eyes. Otherwise, the retinas appeared normal.

When examination 2 months later showed an increase in the size of the hyphaema, the patient was admitted for pars plana washout of the blood. During the surgery, a suprachoroidal haemorrhage and a possible retinal detachment was noted. $\mathrm{C}_{3} \mathrm{~F}_{8}$ gas was injected. A large rubeotic vessel on the iris was cauterised. One week postoperatively, examination showed a $20 \%$ hyphaema.

One month postoperatively, however, examination showed a $100 \%$ hyphaema and no view of the fundus. Ultrasonography was performed and showed no obvious retinal detachment, but revealed thickening of the peripapillary retina and choroid with vitreous membranes extending to this region. At 3 months the hyphaema cleared completely but there was still a poor view of the fundus secondary to persistent anterior vitreous haemorrhage. At 4 months, the patient underwent a vitrectomy to remove the persistent vitreous haemorrhage. A thick fibrin-like membrane was noted in the vitreous. The retina was attached in all quadrants with the exception of the inferonasal quadrant where the retina was organised and showed contraction anteriorly. Along the superior arcade 
and across the macula, there was a fibrous membrane extending to the disc. This membrane was severed at several points to relieve all the traction. The postoperative course was stable, but at 4 months, the examination showed the persistence of the inferonasal retinal elevation and the macular epiretinal membrane. Vision though remained stable at $6 / 120$.

\section{CASE 5}

A 63-year-old man was referred for management of keratouveitis in the right eye. Ocular history was significant for background diabetic retinopathy in the right eye, proliferative diabetic retinopathy, status post scatter photocoagulation in the left eye, and no history of rubeosis in either eye. The patient has had diabetes mellitus for $\mathbf{1 7}$ years, congestive heart failure, but no carotid abnormalities. He was taking insulin, captopril, procainamide, and frusemide.

On presentation, visual acuity was hand motions in the right eye and 6/24 in the left eye. Pupillary responses and motility were within normal limits. Applanation tensions were 18 and $12 \mathrm{~mm} \mathrm{Hg}$ in the right and left eyes. The right eye showed diminished corneal sensation compared with the left. Anterior segment examination of the right eye showed 4+ ciliary flush, a large geographic corneal epithelial defect with dendritic figures that stained with fluorescein and mild stromal oedema with folds in Descemet's membrane. About 20 large brownish grey keratic precipitates, with $4+$ cell and flare were noted in the deep anterior chamber. Lens showed moderate nuclear sclerosis. Gonioscopy showed open angles with no synechiae or rubeosis. Anterior segment examination of the left eye was notable only for moderate nuclear sclerosis. Dilated fundus examination showed clear vitreous in both eyes and no proliferative activity in either fundi. Both fundi showed scattered microaneurysms and haemorrhages with laser scars in the periphery of the left eye.

The patient was started on topical trifluridine every 2 hours for 5 days. At the end of 5 days, the epithelial lesions had healed, but stromal oedema, keratic precipitates, and anterior chamber inflammation persisted and the intraocular pressure (IOP) rose to 22. To control this inflammation and IOP, topical prednisolone acetate with betaxolol $0.5 \%$ was started. One week later, the IOP had fallen to 16 , the stromal oedema had resolved, but there was some stromal scarring. There were fewer keratic precipitates and cells in the anterior chamber. With clinical improvement, topical trifluridine and prednisolone acetate were both slowly tapered and betaxolol was discontinued. Four weeks later, on once a day trifluridine and prednisolone (Fig 2), the patient developed more pain and redness. Visual acuity was $6 / 120$ and anterior segment examination showed a $0.5 \mathrm{~mm}$ hyphaema and $1+$ red blood cells in the anterior chamber. Although the corneal scar remained, there were no epithelial defects, folds or keratic precipitates. No iris rubeosis was seen, but synechiae to the lens were noted. In response, topical prednisolone acetate was increased and homa-

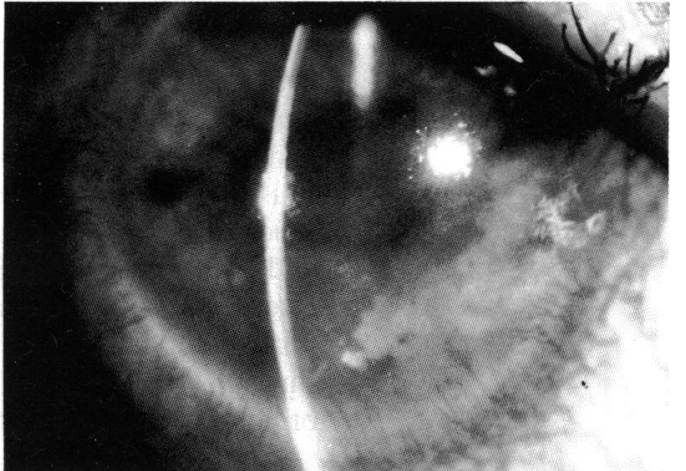

Figure 2 Anterior segment photograph of right eye, case 5.

tropine $5 \%$ was added. Over 8 days, the hyphaema resolved. During the 2 months of follow up, rubeosis was never detected. Vision remained at $6 / 120$ owing to the corneal scarring and cataract. Fluorescein angiography performed on the right eye showed no proliferative changes or significant capillary drop out.

\section{Discussion}

Spontaneous hyphaema can result from a variety of causes (Table 1), but it is rarely seen as a complication of anterior uveitis. We report five patients, all with anterior uveitis, who developed hyphaema: a 44-year-old man with recurrent iritis, rubeotic vessels, and Reiter's syndrome; a 41-year-old woman with recurrent anterior inflammation, iris rubeosis, and juvenile chronic arthritis; a 25-year-old man with iritis and ankylosing spondylitis; a 10 -year-old boy with recurrent idiopathic anterior uveitis and abnormal iris vessel; and a 63-year-old man with herpes zoster keratouveitis. Cases of hyphaema with iritis have been described with several uveitic entities: gout, rheumatoid arthritis, erythema nodosum, gonococcal infections, herpes simplex infections, ${ }^{1}$ and Bechçet's disease, ${ }^{2}$ but the literature lacks sufficient descriptions of uveitis associated haemorrhage to determine the nature, incidence, or outcome of this complication.

Duke-Elder and Perkins noted that in uveitis, severe inflammation can lead to the formation of petechial haemorrhages and haemorrhagic iritis. ${ }^{1}$ There are three mechanisms by which a hyphaema may result from iritis: (1) leakage from delicate rubeotic vessels: (2) damage to vessels from vasculitis; and (3) increased diapedesis. Rubeosis is often associated with posterior segment disease, but rubeosis can

Table 1 Causes of hyphaema ${ }^{10}$

$$
\begin{aligned}
& \text { Trauma } \\
& \text { Post-surgical } \\
& \text { Congenital anomalies } \\
& \text { Persistent hyperplastic primary vitreous } \\
& \text { Persistent pupillary membrane } \\
& \text { Uveitis } \\
& \text { Rubeosis } \\
& \text { Tumours } \\
& \text { Melanomas } \\
& \text { Juvenile xanthogranuloma } \\
& \text { Iris haemangiomas } \\
& \text { Haemorrhagic diathesis } \\
& \text { Leukaemia } \\
& \text { Haemophilia } \\
& \text { Drug } \\
& \text { Anti-platelet agents } \\
& \text { Anti-coagulants }
\end{aligned}
$$


occur with anterior segment inflammation alone. Perry et al reported a case of histopathologically confirmed rubeosis in Fuch's heterochromic iridocyclitis. ${ }^{3}$ Vasculitis can disrupt endothelial cells and their basement membrane causing hyphaema by the leakage of serum and blood. Finally, hyphaema may result from acute and chronic inflammation alone through an intensification of diapedesis. Diapedesis can be a normal process where red blood cells pass through vessel walls following marked congestion or behind migrating white blood cells. ${ }^{4}$

In three of the five patients in our series (cases $1,2,4)$, hyphaema was associated with rubeotic vessels on the iris. In these cases, the mechanism of hyphaema formation is probably leakage from these incompetent vessels. In the two patients (cases 2,3) who were taking NSAIAs, the antiplatelet activity of these drugs may have contributed to hyphaema formation, but NSAIAs are often used without complication by anterior chamber haemorrhage. In one patient (case 2) who was taking NSAIAs, iris rubeosis was also present; the haemorrhage may have been due to the combination of the two factors. The last patient (case 5) was not taking NSAIAs and did not have rubeotic iris vessels. Hyphaema formation in this one case was idiopathic. Screening coagulation studies should have been performed in the examination of our cases, but none of our patients demonstrated any signs or symptoms of systemic bleeding diathesis.

Reiter's syndrome is characterised by arthritis, conjunctivitis, and urethritis. Conjunctivitis is the most common ocular complaint. The conjunctivitis is usually self remitting and characterised by a watery discharge. Iritis occurs in $3-12 \%$ of patients. It is usually mild and nongranulomatous. Keratitis can also occur, but is less common and is characterised by subepithelial and anterior stromal infiltrates. ${ }^{5}$ Case 1 appears to be the first report of the association between Reiter's associated iritis, rubeosis, and hyphaema.

Patients with certain subtypes of juvenile chronic arthritis (JCA) are prone to develop iritis. Girls with pauciarticular arthritis whose serum is negative for rheumatoid factor and positive for antinuclear antibody are at the highest risk for chronic iridocyclitis. ${ }^{6}$ Boys with pauciarticular arthritis who are HLA-B27 positive may develop recurrent anterior uveitis.

Flare predominates in the chronic uveitis of JCA with varying numbers of white blood cells in the anterior chamber. This chronic inflammation can lead to band keratopathy, posterior synechiae, cataract, hypotony, glaucoma, and rarely vitritis with macular oedema. Hyphaema with rubeosis and classic JCA has not been previously reported in the literature. However, in the Russian literature there is one report of a child with hyphaema and infectious non-specific polyarthritis.?

The ophthalmic findings associated with ankylosing spondylitis include both iritis and conjunctivitis. Ocular involvement occurs in about $25 \%$ of patients with arthritis. ${ }^{8}$ Severe inflammation with fibrin clot in the anterior chamber and posterior synechiae are relatively common. Although case 3 was taking NSAIAs, the patient did not demonstrate any clinical signs of bleeding diathesis. The mechanism of hyphaema is the chronic inflammation from ankylosing spondylitis.

Herpes simplex keratitis is a common disease. Uveitis usually develops in association with stromal disease, but it may also occur with the onset of epithelial disease. Secondary glaucoma often accompanies herpetic uveitis. ${ }^{9}$ In cases of uveitis topical steroids should be started expeditiously. Although mild cases may be self limited, severe and/or recurrent cases can cause scarring and be destructive. These undesirable sequelae often lead to the use of topical steroids before epithelial healing. Case 5 shows features of herpetic keratitis, uveitis, secondary glaucoma, and hyphaema.

Currently, there is little information on the incidence of hyphaema in uveitis. While this study was not designed to address this question, in our experience, the presence of hyphaemas complicating uveitis is not common. Because there are so few reported cases of hyphaema and uveitis, the literature has not been helpful in describing the aetiology or the management of this complication. The current report describes five cases of hyphaema related to anterior uveitis and their outcomes. Four of five cases show resolution with medical management. No hyphaema related complications were observed. In only one case was surgical intervention necessary (case 4). Although this experience may not be universal, the current series provides evidence that conservative medical management usually suffices. Needless to say, medical management should include the search and examination for other causes for haemorrhage (Table 1).

In summary, hyphaema is an uncommon complication of anterior uveitis. In the current report, hyphaema was observed in patients with Reiter's syndrome, juvenile chronic arthritis, ankylosing spondylitis, and herpes simplex ophthalmicus. In three patients iris rubeosis was noted and in two patients there was NSAIA use. The clinical course of the five reported cases showed that medical management may be all that should be performed for this complication.

1 Duke-Elder S, Perkins ES. The clinical signs and symptoms of uveitis. In: Duke-Elder, ed. System of ophthalmology, Volume IX. St Louis: Mosby, 1966: 133.

2 Mamo JG. Baghdassarian A. Behçet's disease. Arch Ophthalmol 1971; 71 : 38-48.

3 Perry HD, Yanoff M, Scheie HG. Rubeosis in Fuchs' heterochromic iridocyclitis. Arch Ophthalmol 1975; 93: 337-9.

4 Robbins SL, Cotran RS, Kumar V. Fluid and hemodynamic derangements. In: Pathologic basis of disease. Philadelphia: derangements. In:

5 Lee DA, Barker SM, Su WPD, Allen GL, Liesgang TJ, Illstrup DM. The clinical diagnosis of Reiter's syndrome. Ophthalmology 1983; 93: 350-6.

6 Cassidy JT, Levinson JE, Bass JC, Baum J, Brewer EJ, Fink $\mathrm{CW}$, et al. A study of classification criteria for a diagnosis of juvenile rheumatoid arthritis. Arthritis Rheum 1986;29: 274 81.

7 Komarova NI. Involvement of the eye in a child with infectious nonspecific polyarthritis. Voprosy Okhrany Materinstva I Detstva 1968; 13: 75-6.

8 Brewerton DA, Caffrey M, Nicholls A, Walter D, Oates JK, James DC. Ankylosing spondylitis and HLA-B27. Lancet 1973; i: 904-7.

9 O'Connor GR. Recurrent herpes simplex uveitis in humans. Surv Ophthalmol 1976; 21: 165-70.

10 Yanoff M, Fine B. Ocular pathology. A textbook and atlas. 3rd ed. Philadelphia: Harper and Row, 1989; 428. 